\title{
MMI Structures Covered by Bromocresol Purple for Ammonia Detection
}

\begin{abstract}
A. SzEWCZUK* AND M. BŁAHUT
Department of Optoelectronics, Silesian University of Technology, Akademicka 2, 44-100 Gliwice, Poland

In the paper examinations of a lightguide sensor on the base of gradient index multimode interference structures are presented. Investigated structures were fabricated in potassium-sodium ion exchange process. Multimode interference sensor was designed for ammonia detection. Bromocresol purple covering the multimode section was used as a sensing layer. Numerical calculations were performed with the use of modal analysis and beam propagation method to optimise sensor geometry and sensing layer thickness. Experimental results show the possibility of ammonia detection from the concentration of tens ppm.
\end{abstract}

DOI: $10.12693 /$ APhysPolA.124.570

PACS: 42.79.Gn, 07.07.Df

\section{Introduction}

Optical waveguide sensors, in comparison to their electrical counterparts, have many advantages. The most important of them is resistance to electromagnetic interference and a very fast response. Based on modulation and demodulation processes sensors can be classified into intensity, phase, frequency, polarization sensors. The most of phase sensors are based on conventional interferometers configuration such as the Mach-Zehnder, Michelson, Fabry-Perot, Sagnac [1]. Commercial potential is possessed by devices using simple transducers made in low cost uncomplicated production process, which characterize high reliability of operation. Multimode interference (MMI) structures made in ion exchange process are an example of such devices. The basis of their operation is the phenomenon of input field imaging for the certain propagation length. This characteristic property of these structures can be used in many different ways. The most popular is the use of these optical elements as waveguide splitters [2]. In papers [3-5] we have shown the possibility of the use of MMI structures in gas sensor technology. This problem was also considered by other authors [6-8]. In the paper [6] there has been proposed an optical temperature sensor based on MMI structures in silicon. Temperature changes cause changes in the refractive index of the interference section. The studies of interference structures made in planar waveguides by sol-gel method $[7,8]$ were concerned with technological and experimental research of water vapor sensor. There were also presented the numerical simulations of the interference gas sensor operation.

In this work, examinations of MMI structures made by potassium-sodium ion exchange are presented. Multimode gradient index sections are covered by bromocresol

*corresponding author; e-mail: artur.szewczuk@polsl.pl purple layers that highly react with ammonia. Bromocresol purple is characterized by refractive index higher than the waveguide core refractive index and during ammonia gassing this material becomes highly absorbing for considered wavelength. Theoretical analysis was based on modal attenuation calculations and beam propagation method (BPM) simulations of light propagation. There are also presented experimental results of sensor reaction with ammonia for different gas concentration.

\section{Optical structures}

Multimode interference structures consist of three sets of waveguides: singlemode input and output waveguides and multimode section placed between them. When exciting a multimode waveguide, we can observe the matching of input field to the modal fields of the waveguide, and then the interference of the excited waves. In effect of the intermodal interference, a so-called self-imaging of the input field is taking place. As a result of this effect, the input field is reproduced in direct, reflected and multiple images [2].

The basis of the research described in this paper is a gradient index MMI structure (Fig. 1) with multimode section length relating to the length of the input field image formation. Multimode section is covered with material which changes its optical parameters (refractive index and extinction coefficient) when exposed to appropriate gaseous environments. The changes of external physical parameters lead to the changes of propagation conditions of light, and this effect is easily observable in the output signal. The coating parameters changes have an effect on light propagation condition in structure and can be seen as the output signal variation.

In this configuration, there are several factors responsible for changes in the registered output signal. Variations of coating layer refractive index influence the mode properties of the multimode waveguide, which results in the input field image position and the output signal value. In the case of the absorption layer the refractive index 


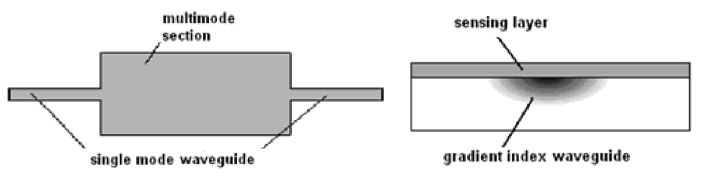

Fig. 1. Model of MMI sensor.

(a)

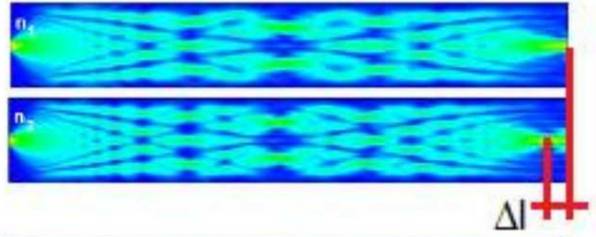

(b)

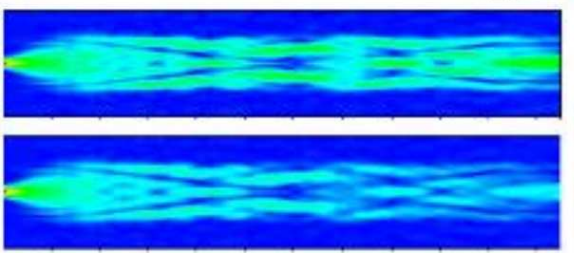

Fig. 2. BPM simulations in MMI sensor. (a) Predominance of phase effect, (b) predominance of absorbing effect.

changes have also influenced the modal attenuation value by changing the shape of the modal field distribution, which leads to a reduction or increase of the amount of light penetrating the absorbing sensing layer. This effect is different for different modes. Depending on the properties of a sensor layer - its refractive index and extinction coefficient - there is observed the predominance of the first phase (Fig. 2a) or second absorbing (Fig. 2b) effect. In this paper, we focus on the absorbing effect occurring in the sensing layers used to measure the gas concentrations.

\section{Material properties}

The investigated MMI sections are produced in the $\mathrm{K}^{+}-\mathrm{Na}^{+}$ion exchange process in the time of $0.75 \mathrm{~h}$ and temperature of $400{ }^{\circ} \mathrm{C}$ in borosilicate glass. The distribution profile of refractive index of MMI section obtained in the diffusion process is calculated numerically from the nonlinear diffusion equation [9].

The multimode waveguide fabricated in ion exchange process was covered by bromocresol purple layer by spin coating. This material is characterized by high refractive index amount to 1.691 and in the case of ammonia gassing it becomes highly attenuating the light for considered wavelength of $635 \mathrm{~nm}[10]$.

\section{Numerical analysis}

The evanescent field of modes propagating in multimode waveguide penetrates the absorbing sensor layer. Due to relatively high value of the sensor layer extinction coeffcient, light can be strongly attenuated just at short propagation distance [5]. To determine modal attenuation coefficients we transform $2 \mathrm{D}$ problem to $1 \mathrm{D}$ using the effective index method. Analysed 1D layer structure is shown in Fig. 3. Refractive index profile of structure for equivalent refractive index distributions, obtained for each [5] mode of MMI structure by the effective index method is presented in Fig. $4 \mathrm{~b}$.
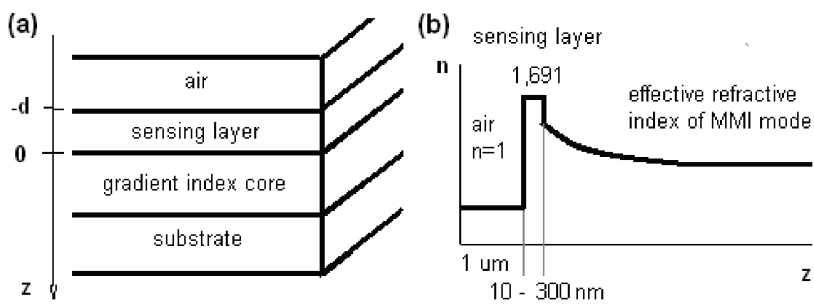

Fig. 3. Configuration of optical structure: (a) layers configuration, (b) refractive index profile.

The formula on modal attenuation coeffcient $\gamma_{m}^{\mathrm{TE}}$ of TE modes of the order $m$ can be expressed by equation [11]:

$$
\gamma_{m}^{\mathrm{TE}}=\frac{2 k_{0} n \kappa}{N_{m}^{\mathrm{TE}}} \frac{\int_{-d}^{0} E_{y}^{2} \mathrm{~d} z}{\int_{-\infty}^{\infty} E_{y}^{2} \mathrm{~d} z},
$$

where $k_{0}$ denotes the wave vector in vacuum, $n$ and $\kappa$ are, respectively, the refractive index and extinction coefficient of the sensor layer, $d$ denotes the sensor layer thickness, $N_{m}^{\mathrm{TE}}$ are the effective indices of TE mode of order $m$. $E$ is the value of electric field.

The modal attenuation is low for very thin sensing layers (Fig. 4). In this case the mode energy is guided mainly in the core and its evanescent part is very small. Together with increase of the layer thickness there also increases the part of mode field which penetrates the cover and the attenuation rapidly grows. For the suitable large thicknesses of the cover the first order mode appears. The attenuation coefficient of this mode increases together with the layer thickness reaching the maximum value, then the evanescent part of field in the cover decreases and attenuation also decreases.

Number of propagating modes in the multimode section is dependent on the width of the section. Each of these modes is characterized by a different value of the attenuation coefficient. Figure 5 shows the dependence of modal attenuation for TE $[0,0]$, TE $[0,1]$ and TE $[5,1]$ modes. Calculations were carried out for $70 \mu \mathrm{m}$ waveguides width, and extinction coefficient equal to 0.00136 . In the case of TE modes $[n, 0]$ attenuation values of each modes are on the same level. More noticeable differences occur in the case of TE modes $[n, 1]$ for the appropriate thickness of a sensor. In this case, there is a possibility to select the configuration for which lower order mode will be stronger attenuated, and the signal will be transmitted only by high order modes with lower attenuation.

Taking into consideration results of mode attenuation, the operation characteristics of MMI sensor are made us- 


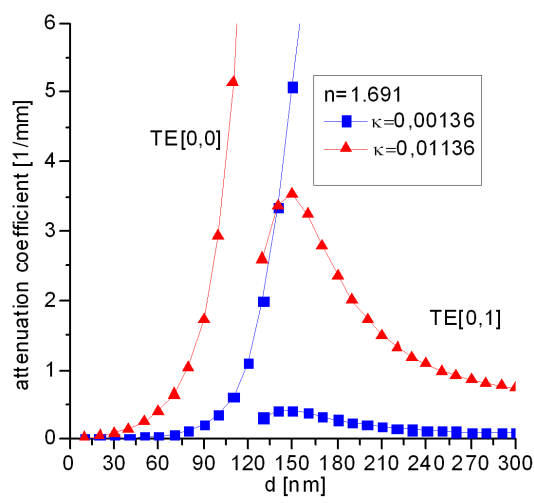

Fig. 4. Attenuation coefficient of core mode in MMI structure as a function of sensor layer thickness for different extinction coefficient $\kappa$ of the layer.

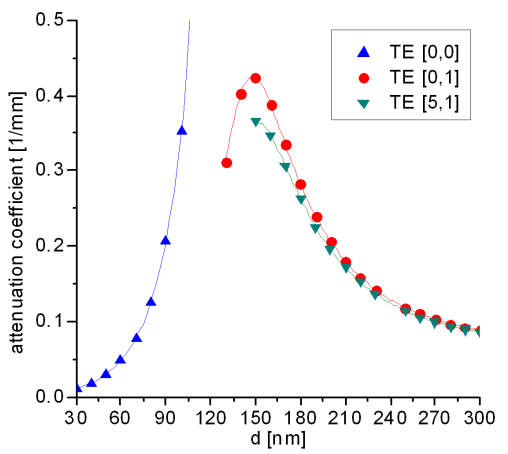

Fig. 5. Attenuation coefficient of mode in MMI structure as a function of sensor layer thickness for different order number TE modes.

ing BPM method for different thicknesses of sensor layers for both polarizations.

Calculations are carried out for MMI section of the width of $30 \mu \mathrm{m}$. Its length, selected to obtain the 1-fold image for TE polarization of the input field, amounts to $2300 \mu \mathrm{m}$. Figure 6 presents the output field amplitude as a function of exaction coefficient of the sensor layer for different values of the layer thicknesses for the TE mode. Thickness of sensing layer is the key parameter which

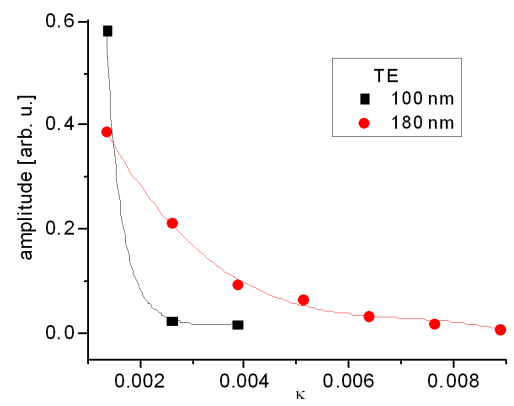

Fig. 6. Output field amplitude as a function of the optical and geometrical parameters of the sensing layer for the different sensing layer thicknesses. affects the operation characteristics of optical sensor with high refractive index absorption cover.

\section{Experimental results}

In order to prepare MMI structures, thin layers of aluminum were applied in a physical sputtering process from the gas phase (PVD method) to cleaned glass substrate from BK-7 glass. Then, in the aluminum layer, the geometry of performed structure was mapped by photolithography process. The ion exchange process of potassium ions from $\mathrm{KNO}_{3}$ salt has been carried out through the prepared mask in time of $1 \mathrm{~h}$ at $400^{\circ} \mathrm{C}$. These parameters ensure a single-mode waveguide fabrication in the direction perpendicular to the surface (one-dimensional interference). Due to the small size of the output waveguides and the method of excitation from the front of the glass, it was important to obtain high-quality edge without any micro laceration. Glasses were trimmed with diamond blade and then after the process of grinding polished with diamond pastes in special holders. Sensor layer was applied by spin coating method. In the experimental studies structure covered with bromocresol purple of $37 \mathrm{~nm}$ thickness was used. The singlemode input fiber was connected to the structure.

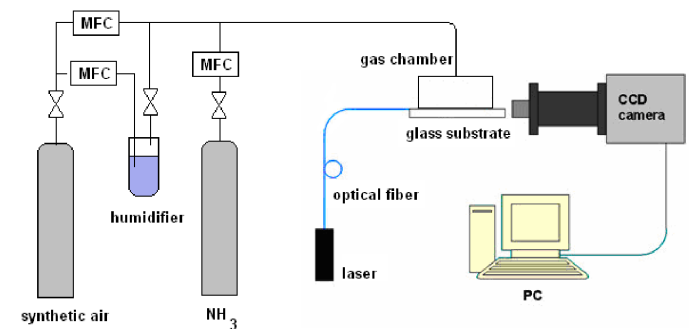

Fig. 7. The experimental stand.

The experimental stand for testing of MMI structures under gassing has been designed and tested (Fig. 7). The single-mode input fiber was excited by laser with wavelength of $0.635 \mu \mathrm{m}$. Then by suitably precise aligning system, the signal was adjusted to the structure. The signal at the output of the structure was recorded using a sensitive CCD camera with an optical system providing a sufficiently high magnification. Appropriate gas concentrations were achieved by mass flow controller (MFC). The research was carried out under gassing in a specially prepared measuring chamber pressed against the glass surface. The inlet and outlet of gas was held by fast-coupling connectors on the top of the chamber. The chamber was pressed through the central part to the glass substrate. The input and output waveguides were uncovered which allowed us to record the output signal by the camera and to excite structure through the fiber.

The amplitude of the light intensity registered by the camera at the end of a single-mode output waveguide was taken as the output signal of the sensor. Measurements 
were made at room temperature in the time intervals in which the structure was gassed alternately with ammonia at various concentrations and with synthetic air.

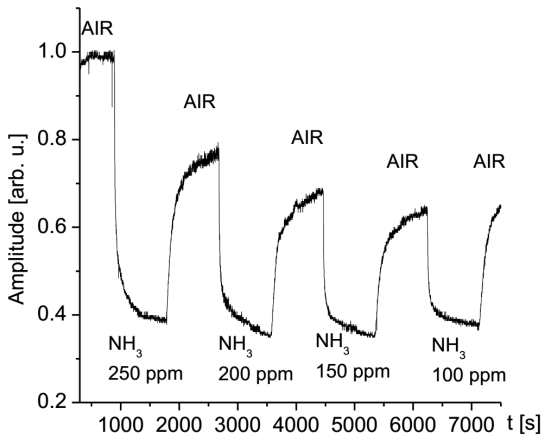

Fig. 8. Output field signal for the different concentration of ammonia in the range from $250 \mathrm{ppm}$ to $100 \mathrm{ppm}$.

Figure 8 shows the value of the measurement signal for different concentrations of ammonia. In this trial, the concentration was varied in the range from $250 \mathrm{ppm}$ to $100 \mathrm{ppm}$. Gassing cycles interval was equal to $15 \mathrm{~min}$. Based on the prepared characteristics it can be seen that apart from the value of the ammonia concentration, the measurement signal during the gassing decreases always to the same value. Furthermore, the signal value during air detoxification cycle does not return to the initial level. In this case, the applied gas concentrations were so high that their further increase did not change the physical and chemical properties of material.

These presumptions were confirmed in characteristics, which represent the value of the measurement signal for the structure gassed with ammonia at low concentrations (in the range of 50 to $10 \mathrm{ppm}$ ) and longer time intervals (Fig. 9). The sensor response was different for different concentrations. Furthermore, longer time cycles caused that the sensing material was detoxified during the cycle in which the structure is exposed to synthetic air.

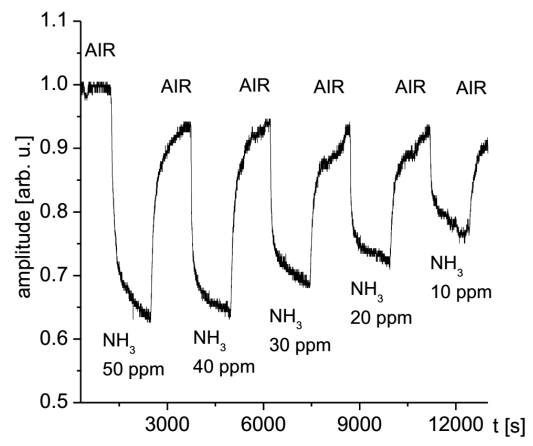

Fig. 9. Output field signal for the different concentration of ammonia in the range from $50 \mathrm{ppm}$ to $10 \mathrm{ppm}$.

Based on these characteristics, dependence of the output signal percentage change as a function of the ammonia concentration was prepared (Fig. 10). It can be seen that even for not large concentrations of ammonia at room temperature there was obtained a clear response of the sensor.

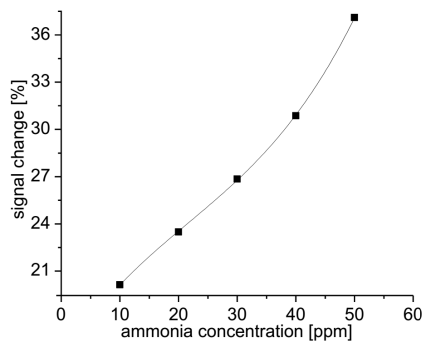

Fig. 10. Percentage sensor response for different concentrations of ammonia.

\section{Conclusions}

In this paper the results of research on the conception of optical fiber sensor based on gradient index multimode interference structure were presented. Experimental works were carried out for the waveguide coated with a thin sensors layer of a $37 \mathrm{~nm}$ thickness. According to the theoretical analysis it is preferable to use slightly thicker layer, but it is connected with the technology problem of the application of thicker layers bromocresol purple. Nevertheless, examined optical systems were characterized by high sensitivity and fast response to changes in the gaseous environment. The result can be improved by applying a higher work temperature of the sensor.

\section{Acknowledgments}

Authors received a grant under the project DoktoRIS - Scholarship program for innovative Silesia co-financed by the European Union under the European Social Fund.

\section{References}

[1] P.V. Lambeck, Meas. Sci. Technol. 17, R93 (2006).

[2] M. Błahut, D. Kasprzak, Opt. Appl. 34, 574 (2004).

[3] A. Szewczuk, M. Błahut, Acta Phys. Pol. A 118, 1254 (2010).

[4] A. Szewczuk, M. Błahut, W. Pyka, Acta Phys. Pol. A 118, 1250 (2010).

[5] A. Szewczuk, M. Błahut, Acta Phys. Pol. A 120, 740 (2011).

[6] A. Irace, G. Breglio, Opt. Expr. 11, 2807 (2003).

[7] K. Kribich, R. Copperwhite, H. Barry, B. Kolodziejczyk, J.M. Sabattie, K. O'Dwyer, B.D. MacCraith, Sensors Actuat. B 107, 188 (2005).

[8] T. Mazingue, R.K. Kribich, P. Etienne, Y. Moreau, Opt. Commun. 278, 312 (2007).

[9] C. Tyszkiewicz, P. Karasiński, R. Rogoziński, Acta Phys. Pol. A 122, 915 (2012).

[10] P. Karasiński, Opt. Appl. 32, 775 (2002).

[11] P. Karasiński, R. Rogoziński, Opt. Commun. 269, 76 (2007). 